based therapy with the bactericidal carbapenem, ertapenem, in patients with CARP. Here, we report on the longer-term outcomes of oral ertapenem in a larger cohort of patients

Methods Patients with CARP were identified retrospectively from a trust-wide Inflammatory Bowel Disease database during the study period (2014-2020). All these patients had undergone faecal coliform sensitivity testing resulting in identification of an ertapenem-sensitive coliform. Clostridioides difficile was excluded in all patients. Modified pouchitis disease activity index (mPDAI) and stool frequency were recorded both pre- and 12-months post-treatment with oral ertapenem. Twelve-month treatment persistence rates, total treatment duration, and prior antibiotic therapy were also recorded. A paired samples t-test was used to compare pre- and post-ertapanem stool frequency.

Results We included 9 patients with a median age was 50 years (range 27-60), 67\% were male. All patients underwent IPAA for UC. All patients had trialled metronidazole and/or ciprofloxacin prior to faecal coliform sensitivity testing and subsequent oral ertapenem therapy. Median pre-ertapenem mPDAI was $5(n=9$, range 2-8), dropping to 3 at 6-weeks $(n=4$, range $3-5)$ and further dropping to 2 at 12-months $(n=3$, range 1-6). Pre-ertapenem mean stool frequency was 15/24-hours (range 5-24), and fell significantly to 6.5/24-hours (range 3-22) after 12-months of treatment, $\mathrm{p}=0.013$ (patientwise data depicting differences in pre and post treatment daytime and night-time stool frequency are shown in figure 1). Eighty-nine percent of patients persisted with oral ertapenem therapy past 12-months (one patient was lost to follow up). None of the patients experienced any adverse events during the 12-month study period.

CHANGE IN NIGHT-TIME STOOL FREQUENCY AFTER 12-MONTHS ORAL ERTAPENEM TREATMENT

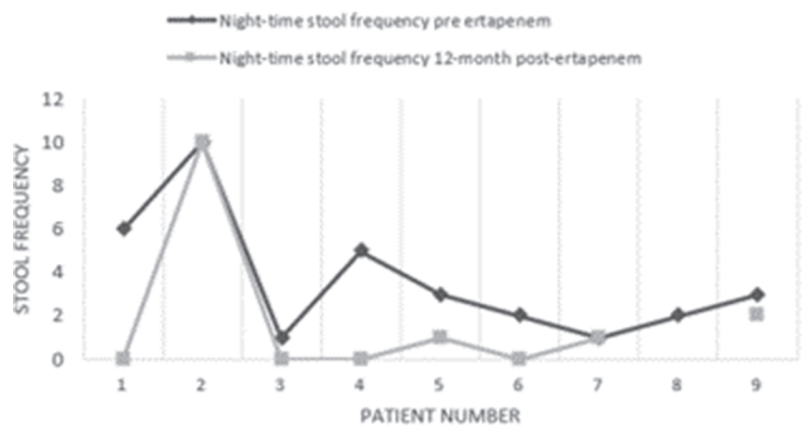

CHANGE IN DAY-TIME STOOL FREQUENCY AFTER 12-MONTHS ORAL ERTAPENEM TREATMENT

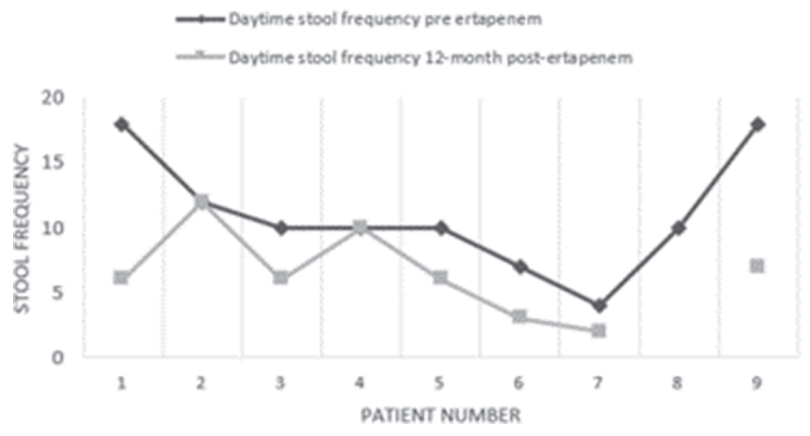

Abstract PM0-43 Figure 1 Change in night-time stool frequency after 12-month oral ertapenem treatment
Conclusion This real-world series of 9 patients with CARP who underwent faecal coliform sensitivity testing further highlights the potential utility of oral ertapenem in treating CARP. There were no reported adverse events and this is compatible with the minimal bioavailability of ertapenem after oral administration.

\section{PMO-44 PYODERMA GANGRENOSUM AND INFLAMMATORY BOWEL DISEASE: A RARE EXTRA-INTESTINAL MANIFESTATION}

${ }^{1}$ Ali El Rida El Masri ${ }^{*},{ }^{2}$ Kotryna Tulabaite, ${ }^{1}$ Bu'Hussain Hayee, 'Lucy Medcalf, ${ }^{1}$ Patrick Dubois, ${ }^{2}$ Sarah Walsh, 'Alexandra J Kent. 'Department of Gastroenterology, King's College Hospital, London, UK; '2Department of Dermatology, King's College Hospital, London, UK

\subsection{6/gutjnl-2021-BSG.183}

Introduction Pyoderma gangrenosum (PG), a neutrophilic dermatosis, is an uncommon extra-intestinal manifestation of IBD. There is paucity of research in the literature identifying key parameters of the disease. In this retrospective study, we describe the cohort in a hospital providing Dermatology and IBD tertiary services, and assess co-presence of the diseases and the disease phenotypes.

Methods Using the electronic patient records, patients who were diagnosed with and/or treated for pyoderma gangrenosum were identified. Key demographics, co-morbidities, and laboratory results were collected, alongside information regarding IBD phenotype and therapeutic interventions.

Results 50 patients across the Trust were identified with PG. The median age was 52 years with $74 \%$ female. Most of the PG lesions fit into the 'typical' category of the disease presentation (70\%), defined as ulcerative lesions on the legs. An atypical presentation is when the disease is present either elsewhere or has bullous/pustular lesions. 50\% PG patients suffered with abdominal symptoms during a PG flare, while only $16 \%$ suffered with joint symptoms. The most common codiagnosis with PG was IBD (42\%), with other disease groups including haematological (14\%), autoimmune diseases (12\%), infectious (6\%), and idiopathic (26\%). Calprotectin levels for the general cohort rose from a baseline median of 109 microgram/gram to a median of 843 during a PG flare (average of 584 and $1153 \mathrm{microg} / \mathrm{g}$ respectively).

The IBD subgroup accounted for $21 / 50$ patients. $50 \%$ of the UC cohort had an extensive phenotype (pancolitis), while $78 \%$ of the Crohn's patients had colonic disease. In the IBD subgroup, most patients were female $(86 \%)$ and the majority of the PG lesions were 'typical' (66.6\%). Faecal calprotectin levels rose from a baseline median $281 \mathrm{ug} / \mathrm{g}$ to a median 1183ug/g during a flare of PG (mean 770 and $1396 \mathrm{ug} / \mathrm{g}$ respectively). The raised faecal calprotectin was seen in both UC and CD, but was more marked in UC. The majority of the IBD cohort received a corticosteroid (85.7\%) and/or another immunosuppressant $(71.4 \%)$ during the PG flare. In $90.5 \%$ of those cases, PG went into remission.

Conclusion Pyoderma gangrenosum is a rare dermatological condition associated with IBD. In our cohort, PG has been more strongly associated with IBD than any other condition and was more common in pan-UC and colonic CD. Faecal calprotectin was higher during a flare of PG compared to the baseline (mean 1396 vs 770, median 1183 vs 281 microg/g), even in those without an IBD diagnosis. Immunosuppressive 
therapy is effective in the majority of patients, whether directed at the IBD or the PG.

\section{PM0-45 FAECAL CALPROTECTIN, AN ALTERNATIVE MARKER TO ESTIMATE CUMULATIVE INFLAMMATORY BURDEN IN ULCERATIVE COLITIS}

Alexander Robertson*, Antonia Churchhouse, Ross Porter, Kathryn Kirkwood Katherine Quiohilag, Gareth-Rhys Jones, Eleanor Watson, Shahida Din. Edinburgh IBD Unit, Western General Hospital, Edinburgh, UK

\subsection{6/gutjnl-2021-BSG.184}

Introduction Long-term Ulcerative Colitis (UC) increases the risk of colonic dysplasia and colorectal cancer (CRC). We aim to establish whether real-world faecal calprotectin (FCP) data can be used to estimate the cumulative inflammatory burden (CIB) and identify those at risk of dysplasia and CRC.

Methods Patients with left sided or extensive UC of $>8$ yrs duration, with $>1$ endoscopy and $>1$ serial FCP value (from 2005) were extracted from the NHS Lothian IBD registry. Patients with PSC were excluded.

CIB scores based on histology $(\mathrm{CIB}(\mathrm{H}))$ or FCP $(\mathrm{CIB}(\mathrm{FCP}))$ were calculated based on the method proposed by Choi et al. ${ }^{1}$ Patients were categorised into three groups; IBD-associated dysplasia and CRC (IBD-D/CRC $\mathrm{n}=15)$, only sporadic adenomas $(n=29$, excluded from further analysis) and patients who did not develop any type of dysplasia $(n=220)$.

To give a more accurate estimation of cumulative inflammation FCP levels were defined as low $(n=73)$ or high $(n=162)$. High $\mathrm{CIB}(\mathrm{FCP})$ is equivalent to 5 yrs of a continuous FCP value of $\geq 250 \mu \mathrm{g} / \mathrm{g}$, a surrogate marker of chronic active inflammation.

Results A defined cohort of 264 patients (146 males), with a median age 36 (IQR 27.1-46.9) were included.

Using the $\mathrm{CIB}(\mathrm{H})$ score, patients with no dysplasia $(n=220)$ had a median score of 4.7 (IQR 2.7-7.9), compared with patients with IBD-D/CRC $(n=15)$ who had a score of 5.4 (3.5-8.2) $(\mathrm{p}=0.4405$, unpaired two-tailed t-test).

The median CIB (FCP) score for patients with no dysplasia was 1804 (883-3689), compared with patients with IBD-D/ CRC who had a median score of 2256 (1593-3848) $(p=0.4835)$. The correlation between the two types of CIB scores in identifying risk of IBD-D/CRC was weak (Spearman's rho $=0.296(p<0.001)$. In this cohort neither score was able to predict IBD-D/CRC.

To give a more accurate estimation of $\mathrm{CIB}(\mathrm{FCP})$ the rates of IBD-D/CRC were stratified by FCP levels over time; 73 patients (31.1\%) had low $\mathrm{CIB}(\mathrm{FCP})$ and 162 (68.9\%) had a high $\mathrm{CIB}(\mathrm{FCP})$. Using this model, $1 / 73(1.4 \%)$ in the low CIB (FCP) group and 14/162 (8.6\%) in the high group had IBD$\mathrm{D} / \mathrm{CRC}(\mathrm{p}=0.0417$, Fishers Exact Test), figure 1. Figure 1: low/high CIB(FCP)

Conclusions This uncontrolled cohort study suggests that serial faecal calprotectin measurements can be used to estimate the cumulative inflammatory burden. Patients with chronic UC who have a high CIB (FCP) score were more likely to develop IBD-D/CRC, but there was only weak correlation between $\mathrm{CIB}(\mathrm{H})$ and $\mathrm{CIB}(\mathrm{FCP})$. Further data is required to validate our findings.

\section{REFERENCE}

1. Choi CHR, al Bakir I, Ding NSJ, et al. Cumulative burden of inflammation predicts colorectal neoplasia risk in ulcerative colitis: A large single-centre study. Gut Published Online First: 2019. doi:10.1136/gutjnl-2017-314190

\section{PMO-46 HEALTH-RELATED QUALITY OF LIFE IN PATIENTS WITH CHECKPOINT INHIBITOR ENTEROCOLITIS}

${ }^{1}$ Melissa Torkizadeh*, 1,2,3 Hajir Ibraheim, 1,2 Shiva Radhakrishnan, ${ }^{3}$ James Larkin, 1,2William Howson, ${ }^{1,2}$ Horace Williams, ${ }^{1,2}$ Lucy Hicks, ${ }^{4}$ Calum D Moulton, ${ }^{1,2,3}$ James L Alexander, ${ }^{1,2,3}$ Nick Powell. ${ }^{1}$ Imperial College School Of Medicine, London, UK; ${ }^{2}$ Imperial College Healthcare NHS Trust, London, UK; ${ }^{3}$ The Royal Marsden NHS Trust, UK; ${ }^{4}$ Department of Psychological Medicine, King's College London, London, UK

\subsection{6/gutjnl-2021-BSG.185}

Introduction Immune checkpoint inhibitors (CPI) have transformed the treatment of many advanced cancers but cause immune related adverse events including enterocolitis (CPI-E). The conventional inflammatory bowel diseases ulcerative colitis (UC) and Crohn's disease (CD) are associated with unfavourable health-related quality of life (HRQoL) outcomes, but there are currently no data on HRQoL in the setting of CPI-E. This study aimed to investigate HRQoL in CPI-E.

Methods A prospective study was conducted across two London hospital trusts between February-April 2021. UC, CD and CPI-E patient cohorts were recruited from outpatient clinics and the biologic infusion unit. Disease activity was assessed using non-invasive scoring systems: modified-Partial Mayo Score (m-PMS), modified-Harvey Bradshaw Index (m-HBI), Simple Crohn's and Colitis Activity Index (SCCAI) and Common Terminology Criteria for Adverse Events (CTCAE). HRQoL outcomes were assessed using validated questionnaires: Patient Health Questionnaire-8 (PHQ-8), Generalised Anxiety Disorder-7 (GAD-7), IBD-Questionnaire (IBD-Q) and Multidimensional Assessment of Fatigue (MAF).

Results Seventy-five patients (33 CD, 21 UC, 21 CPI-E) were recruited. $33 \mathrm{CD}$ patients (100\%) and 20 UC patients (95.2\%) were receiving biologic therapy. Thirteen CPI-E patients (61.9\%) received Anti-PD1/PDL1 monotherapy and (38.1\%) received combination anti-PD1 and anti-CTLA-4 therapy. Twenty-four CD patients (72.7\%), 11 UC patients (52.4\%) and 16 CPI-E patients (76.2\%) were shielding due to the COVID-19 pandemic. Using m-PMS, m-HBI, SCCAI and CTCAE, $>80 \%$ in each of the three cohorts were either classed as being in remission or having mild disease activity. Three CPI-E patients (14.3\%) had moderate depression (PHQ$8 \geq 10$ ) and a further $9(42.9 \%)$ had mild depression (PHQ-8 score 5-9). Nine CPI-E patients (42.9\%) had significant fatigue (MAF score $\geq 30)$ and $6(28.6 \%)$ had mild or moderate anxiety (GAD-7 $\geq 5)$. There were no significant differences in PHQ-8, GAD-7, IBD-Q and MAF between CPI-E, CD and UC patients, suggesting comparable levels of psychological morbidity in the three groups. Significant correlations were found between CPI-E disease activity and IBD-Q and GAD-7 scores.

Conclusion Our study suggests that psychological morbidity in CPI-E is common and comparable to rates in CD and UC, even in the setting of clinical remission. Clinicians should be aware of this complication and take a holistic approach to this patient group. 